\title{
Asociación entre el exceso de peso (sobrepeso y obesidad) y la utilización de edulcorantes no calóricos en la dieta diaria en un sector de la ciudad de Cartagena de Indias (Colombia) 2016
}

\section{Lara G, Ramos E, Ruidiaz K, Arrieta J}

MSc(c), Esp. Investigación clínica. Director del Grupo de Investigación en Nutrición y Metabolismo Humano Categoría C Colciencias. Universidad del Sinú seccional Cartagena. Colombia. MSc. Salud Pública. Coordinador de investigaciones Posgrado. Docente Investigador. Grupo de Investigación en Nutrición y Metabolismo Humano Categoría C Colciencias. Universidad del Sinú seccional Cartagena. Colombia.

MSc. en Enfermería. Director del grupo de investigación Cuidado de la Vida y la Salud. Universidad del Sinú seccional Cartagena. Colombia.

IMEng. Ingeniería de software. Docente Investigador. Director Escuela de Ingeniería de Sistemas. Universidad del Sinú seccional Cartagena. Colombia.

Fecha de recepción: 13/03/2017

Fecha de aceptación: 26/07/2017

\section{Resumen}

$\mathrm{E}$ studios realizados en la ciudad de Cartagena de Indias hacia el 2014, evidenciaron una elevada prevalencia de sobrepeso y obesidad, en comparación con otras regiones de Colombia; al 62\% de la población actual de Cartagena (un $41 \%$ de sobrepeso y un $21 \%$ de obesidad). Sin embargo, a pesar de esta "epidemia", existen pocas investigaciones en la ciudad encaminadas al estudio de factores protectores frente a la enfermedad.

Objetivo: Establecer la asociación entre el exceso de peso (IMC $\geq 25$ ) versus la utilización de edulcorantes no calóricos en la dieta diaria de habitantes de un sector de la ciudad de Cartagena de Indias, Colombia.

Metodología: Se realizó un estudio observacional-transversal, cuya muestra involucró a más de 88 viviendas (470 participantes) de la Unidad de Gobierno No.1 de la ciudad de Cartagena de Indias compuesta por barrios de los estratos 4 al 6 que, de acuerdo a los antecedentes del estudio, tienen mayor conocimiento y acceso a los edulcorantes. Para el diagnóstico de la enfermedad (sobrepeso y obesidad) personal entrenado y con instrumentos debidamente calibrados evaluó los parámetros antropométricos. Posteriormente, se aplicó una en- cuesta validada en estudios previos, en cuanto al uso de edulcorantes en la dieta diaria de los participantes. Los resultados se analizaron con el programa estadístico WinEpi utilizando como medida de asociación la "Razón de prevalencias" con un nivel de confianza del 95\%.

Resultados: De acuerdo a los resultados, la Unidad de Gobierno No.1 conformada por los barrios el Laguito, Castillo Grande, Bocagrande, El Centro, La matuna, Getsemaní, San Diego, El cabrero, Marbella, Crespo, Pie de la Popa y Manga presentaron una prevalencia de sobrepeso del 34\%, una prevalencia de obesidad del $9 \%$ y una prevalencia del porcentaje de grasa corporal aumentado del $38 \%$ en el momento del estudio. El $27 \%$ de todos los participantes estuvieron expuestos al consumo diario de edulcorantes.

Conclusiones: De acuerdo con el estudio, los individuos expuestos al uso diario de edulcorante no calórico (130) mostraron entre 3,98 y 13,28 menos probabilidades de presentar sobrepeso u obesidad ( $\mathrm{IMC} \geq 25$ ) que los no expuestos (340). Por lo tanto, se considera que el uso diario de edulcorantes no calóricos en la dieta es un factor protector.

Palabras claves: Obesidad, Sobrepeso, Índice de Masa Corporal, Edulcorante, Dieta, porcentaje de grasa corporal.

\section{Introducción}

Hoy en día, la obesidad se ha convertido en un problema de salud pública por su incremento circunstancial en las últimas décadas $^{(1)}$. Esta predisposición se debe eventualmente a factores genéticos, prenatales, posnatales y al consumo excesivo de alimentos hipercalóricos combinado con el sedentarismo ${ }^{(2)}$. La obesidad ha incrementado a tal punto, que ha rebasado los índices de la desnutrición ${ }^{(3)}$.

Según datos de la Organización Mundial de la Salud (OMS), el sobrepeso está presente en más de mil millones de personas en todo el mundo ${ }^{(4)}$. Este problema no solo afecta a la población adulta, sino también a los menores, tanto en países desarrollados como en países en vía de desarrollo, adquiriendo proporciones epidémicas $^{(5)}$. 
El sobrepeso aumenta sustancialmente no sólo el riesgo de diabetes y de enfermedad cardiovascular, sino también ciertos tipos de cáncer y otras enfermedades altamente prevalentes, de tal manera que la obesidad se ha convertido en una de las principales causas de mortalidad prematura y evitable ${ }^{(6)}$.

En Colombia, estudios realizados por la Fundación Colombiana de Obesidad (FUNCOBES), indican que uno de cada dos colombianos presenta exceso de peso, en especial la población femenina, que representa el $55,2 \%$ de los casos, frente a un $45,6 \%$ en los hombres ${ }^{(7,8)}$.

La falta de actividad física también contribuye a esta problemática; de hecho, solamente un $12 \%$ de la población colombiana cumple con las recomendaciones de actividad física $\geq 150$ minutos a la semana ${ }^{(9,10)}$.

La Encuesta Nacional de la Situación Nutricional en Colombia 2010 (ENSIN) demostró que la prevalencia de obesidad en el departamento de Bolívar se encuentra en un $21,4 \%$, cifra bastante considerable frente a las demás regiones de Colombia ${ }^{(11)}$.

Estos resultados despiertan una gran preocupación por las generaciones futuras y plantean la necesidad de reforzar las estrategias educativas para la promoción de estilos de vida saludables, por parte de los entes territoriales y la academia.

Por otro lado, en regiones con programas exitosos de lucha contra la obesidad, un componente primordial es la formación de hábitos alimentarios saludables a nivel del "núcleo familiar", que es el primer determinante de hábitos y costumbres culturales; al romper ese lazo de generaciones, se reduce la prevalencia de enfermedades crónicas no transmisibles a causa de un inadecuado estilo de vida.

Los actuales programas para la prevención del exceso de peso en Colombia, poco contemplan una real intervención a nivel del núcleo familiar. Por ello, son pocos los estudios que se han diseñado y ejecutado en Colombia, que permitan enfocar los esfuerzos en "factores protectores" dentro de una misma ciudad o región.

Ahora bien, todas las acciones encaminadas a promocionar estilos de vida saludables están relacionadas con una mejoría significativa de las comorbilidades asociadas a este problema de salud ${ }^{(12)}$.

Por ejemplo, ha sido demostrado que incluso pequeñas reducciones de 5 a $10 \%$ del peso corporal, inducen mejorías significativas en las anomalías metabólicas asociadas al sobrepeso o la obesidad ${ }^{(13)}$; se estima que acciones enfocadas a lograr estas pequeñas reducciones tendrían un alto impacto en la población.

Dada la complejidad de esta enfermedad y las graves consecuencias que lleva consigo, es importante llevar a cabo estudios que reconozcan factores protectores que favorezcan o beneficien la disminución del peso corporal.

El presente estudio tuvo como propósito identificar un factor protector en la población cartagenera, como es el uso de un sustituto del azúcar o edulcorante no calórico en la dieta diaria; se trata de un aditivo para los alimentos que tiene mayor efecto en el dulzor que el del azúcar, pero que usualmente tiene menos kilocalorías.

Además, se busca lograr que los cartageneros reconozcan "factores protectores" en su dieta y estilos de vida, lo cual beneficiará a las personas en la mejoría y disminución de los riesgos asociados al exceso de peso, obteniendo beneficios a nivel metabólico y, por ende, una mayor eficacia en la utilización de energía y el control de los niveles de lípidos en el organismo $^{(14)}$. Por otra parte, la adopción de un estilo de vida saludable, dejando de lado el sedentarismo y las comidas no saludables, contribuiría a favorecer la digestión, por la compresión de las grasas en los órganos internos ${ }^{(15)}$.

De igual forma, un cambio en el estilo de vida tendría una incidencia positiva en el descanso, debido que la grasa corporal comprime las vías respiratorias altas impidiendo que la respiración se desarrolle normalmente ${ }^{(16)}$. Finalmente, las medidas de bienestar, salud y descanso incrementan el autoestima del individuo, pues, el hecho de poder controlar los impulsos al comer y de abandonar el sedentarismo, genera sensación de autocontrol que eleva la confianza en sí mismo y favorece las relaciones sociales ${ }^{(17)}$.

En el futuro, el reconocimiento de "factores protectores" podría ser utilizado como una estrategia en toda Colombia y esto contribuirá a disminuir las cifras elevadas de sobrepeso u obesidad en el país, reduciendo la prevalencia de morbimortalidad que actualmente se está incrementando no solo a nivel nacional, sino internacional; y los costos que acarrea para el gobierno en la atención de estas enfermedades ${ }^{(18)}$.

Este estudio podrá llamar la atención de investigadores, para la implementación de otros estudios que permitan identificar más factores protectores, aportando así a la mejora de esta problemática.

\section{Diagnóstico de la enfermedad}

El sobrepeso y la obesidad se refieren a un exceso de peso corporal comparado con la talla, que afecta a nivel mundial tanto a niños, como a adolescentes y adultos, y se encuentra relacionado con malos hábitos en la alimentación, factores genéticos, falta de educación, costumbres alimentarias y falta de ejercicio ${ }^{(19)}$.

La Organización Mundial de la Salud (OMS) define el sobrepeso y la obesidad como "una acumulación anormal o excesiva de grasa que puede ser perjudicial para la salud"(20).

El diagnóstico y la clasificación del sobrepeso y la obesidad se hacen utilizando tablas que expresan valores en percentiles y puntajes $\mathrm{Z}$, con relaciones como el índice de masa corporal (IMC), este es un indicador simple de la relación entre el peso y la talla, creado por el estadístico belga Adolphe J. Quetelet alrededor de 1835; y resulta de dividir el peso de la persona entre la talla (estatura), elevada al cuadrado, es decir: IMC = peso $(\mathrm{kg}) /$ talla, al cuadrado $\left(\mathrm{m}^{2}\right)^{(21)}$. 
La OMS establece los límites y categorías de peso estándar, con base en el IMC, y que son aplicables para toda la población, independientemente de la edad (tabla $\mathbf{1})^{(22)}$.

Por otro lado, el sobrepeso y la obesidad son afectaciones multifactoriales, por lo que obedecen a diferentes mecanismos patogénicos: Hernández $\mathrm{T}^{(23)}$. opina que el factor predominante es la "genética", por la frecuencia con la que se encuentran hijos con sobrepeso y obesos, cuyos padres padecen la misma patología. Sin embargo, resulta difícil establecer el valor que tiene la herencia frente a los hábitos socioculturales, adquiridos en el seno familiar y reafirmados con los años.

Similar a él, Stunkard $\mathrm{A}^{(24)}$. expresa que la obesidad tiene un origen asociado con factores socioculturales, como nutrición, sedentarismo y alteraciones psicológicas; por el contrario, existen quienes consideran que es el resultado de la interacción entre "lo que se trae" y "lo que se adquiere"(24).

\section{Tratamiento de la enfermedad y el uso de Edulcorantes}

Ahora bien, el tratamiento de esta enfermedad se sustenta en 3 pilares fundamentales: el primero, actividad física, el segundo, alimentación balanceada y el último, el estilo de vida del paciente a través de la educación para la salud ${ }^{(25)}$. Debe tenerse en cuenta, que el modo de vida no es más que una forma específica e importante de la actividad humana y el estilo de vida es lo individual, que se toma del modo de vida. De este modo, la actividad humana, al tener un carácter complejo, parte de la acción sociocultural del hombre realizada a través de la actividad psíquica del organismo.

Por esta razón, hay que destacar la importancia de la promoción de estilos de vida saludables, los cuales se podrían describir como el conjunto de acciones comunicativas que se utilizan con el fin de modificar, de forma voluntaria, ciertas conductas o prácticas, que influyen de forma cotidiana en la alimentación, la nutrición y la actividad física de las personas, tratando de mejorar su estado nutricional y de salud, y creando nuevas prácticas con base en cambios de actitud y de comportamiento $^{(26)}$.

En la actualidad, una de estas acciones comunicativas se basa en la promoción de sustitutos del azúcar o "edulcorantes no calóricos", cuyo uso continuo en la industria de alimentos ha sido aprobado en países como los Estados Unidos para ayudar en la pérdida de peso, limitando la ingesta de energía de los consumidores al remplazar azúcar de alta energía o jarabe de maíz por edulcorantes que aportan poca o ninguna energía $^{(27)}$.

Esto le permitiría al consumidor "comer" los mismos alimentos que normalmente consumía diariamente, mientras se pierde peso y evita otros problemas asociados con el consumo excesivo de calorías, logrando igualmente, otros beneficios como el cuidado dental, pues los sustitutos del azúcar no son cariogénicos, ya que no son fermentados por la microflora de la placa dental. Además, el uso de edulcorantes no calóricos artificiales contribuye al mejor control de la glucemia en personas con diabetes que tienen dificultad para regular sus niveles de azúcar sanguínea y les permite disfrutar de una dieta variada mientras controlan su consumo de azúcar. Las personas con hipoglucemia reactiva que producen un exceso de insulina, causando que sus niveles de glucosa sanguínea caigan por debajo de la cantidad necesitada para la función adecuada del organismo y el cerebro, al igual que los diabéticos, deben evitar el consumo de alimentos que aumenten la glucemia y frecuentemente escogen edulcorantes artificiales como una alternativa $^{(28)}$.

La industria de alimentos y bebidas está remplazando de forma creciente el azúcar por edulcorantes no calóricos en muchos productos que tradicionalmente contenían azúcar.

En el Reino Unido, por ejemplo, actualmente es casi imposible encontrar algún refresco que no esté endulzado con edulcorantes. Por lo tanto, no es sorprendente que la industria de alimentos esté promocionando altamente sus productos de "dieta" o "light", promoviendo el movimiento de los consumidores hacia estos productos endulzados artificialmente, que son aún más rentables.

Ahora bien, el Comité Conjunto de Expertos sobre Aditivos Alimentarios de la OMS, la Organización de Alimentos y Agricultura (FAO) y el Comité de Expertos en Aditivos Alimentarios (JECFA), han establecido la siguiente Ingesta Diaria Admisible de edulcorantes naturales y artificiales durante toda la vida (desde la infancia) sin riesgos para la salud (tabla 1).

Tabla 1. Ingesta Diaria Admisible de edulcorantes naturales y artificiales

\begin{tabular}{l|l}
\hline \multicolumn{1}{c|}{ Edulcorante } & \multicolumn{1}{c}{ Ingesta Diaria Admisible } \\
\hline Acesulfame de potasio & $15 \mathrm{mg} / \mathrm{kg}$ de peso corporal por día \\
\hline Aspartame & $40 \mathrm{mg} / \mathrm{kg}$ de peso corporal por día \\
\hline Ciclamato sódico & $11 \mathrm{mg} / \mathrm{kg}$ de peso corporal por día \\
\hline Sacarina & $5 \mathrm{mg} / \mathrm{kg}$ de peso corporal por día \\
\hline Sucralosa & $15 \mathrm{mg} / \mathrm{kg}$ de peso corporal por día \\
\hline Steviosida & $2 \mathrm{mg} / \mathrm{kg}$ de peso corporal por día \\
\hline
\end{tabular}

Fuente: OMS/OPS 2010

Estas recomendaciones han sido adoptadas por muchos países en sus marcos regulatorios, incluyendo Argentina, Chile, Brasil, México, Bolivia y Colombia, a pesar de la controversia acerca de los supuestos riesgos sobre la salud de los edulcorantes artificiales tales como la "sacarina y el aspartame" los cuales en algunos estudios han mostrado que causan tumores cerebrales así como cáncer linfático en animales de laboratorio y sugieren que la sacarina causa cáncer de vejiga en animales de laboratorio. Sin embargo, es poco probable que 
esto afecte a los humanos, pues el mecanismo que podría causar que la sacarina sea cancerígena en los ratones no existe en humanos $^{(29)}$.

Por otra parte vale la pena aclarar que según la clasificación de riesgos del uso de medicamentos en el caso de mujeres embarazadas establecida por la Food and Drug Administration (FDA), la sacarina pertenece a la categoría B y el Ciclamato sódico a la categoría C; por lo tanto, ninguno de ellos presenta total seguridad para uso en embarazadas. El aspartame, al ser fuente de fenilalanina, también está desaconsejado por el riesgo de acumulación fetal de este metabolito. En resumen, no es aconsejable el uso de edulcorantes artificiales en mujeres embarazadas ${ }^{(30)}$.

\section{Materiales y Métodos}

Se realizó un estudio observacional-transversal, cuya muestra involucró 470 participantes residentes de la Unidad de Gobierno No.1 de la ciudad de Cartagena de Indias. Se tuvieron en cuenta como criterios de inclusión a personas de ambos sexos entre los 18 y los 50 años de edad (este rango se estableció teniendo en cuenta estudios de inicio de sarcopenia en adultos) que fueran residentes permanentes en la unidad de gobierno a estudiar y que hubiesen firmado el consentimiento informado del estudio. Los criterios de exclusión fueron: personas que hubiesen ingerido alcohol, o hubiesen realizado ejercicio físico en horas previas a la toma de los datos antropométricos, la presencia de procesos febriles, deshidratación o edema y en periodo menstrual, personas que consumieran medicamentos que pudieran alterar su peso corporal, personas que sufrieran de patologías que afectaran su metabolismo y peso corporal, diabetes o trastornos tiroideos, mujeres en embarazo, personas con algún implante metálico o marcapasos (por eventos adversos a la bioimpedancia), y personas que no cumplieran con los parámetros estimados.

\section{Selección de la localidad a estudiar}

El distrito de Cartagena está dividido político-administrativamente en tres localidades: localidad 1, Histórica y del Caribe Norte, localidad 2, De la Virgen y Turística y localidad 3, Industrial de la Bahía. A su vez, las tres localidades están divididas en quince Unidades Comuneras de Gobierno Urbanas. Para la realización de este estudio se incluyeron participantes de la localidad 1, específicamente de la Unidad de Gobierno No.1, compuesta por barrios de los estratos 4 al 6, que de acuerdo a los antecedentes del estudio tienen mayor conocimiento y acceso a los edulcorantes (figura 1) ${ }^{(15)}$.

\section{Operacionalización de las variables e instrumentos para la recolección de datos}

Para la recolección de los datos de exceso de peso (enfermos) se utilizó un registro que incluyó la fórmula de índice de
Figura 1. Estratificación de la ciudad de Cartagena a través de unidades comuneras de gobierno

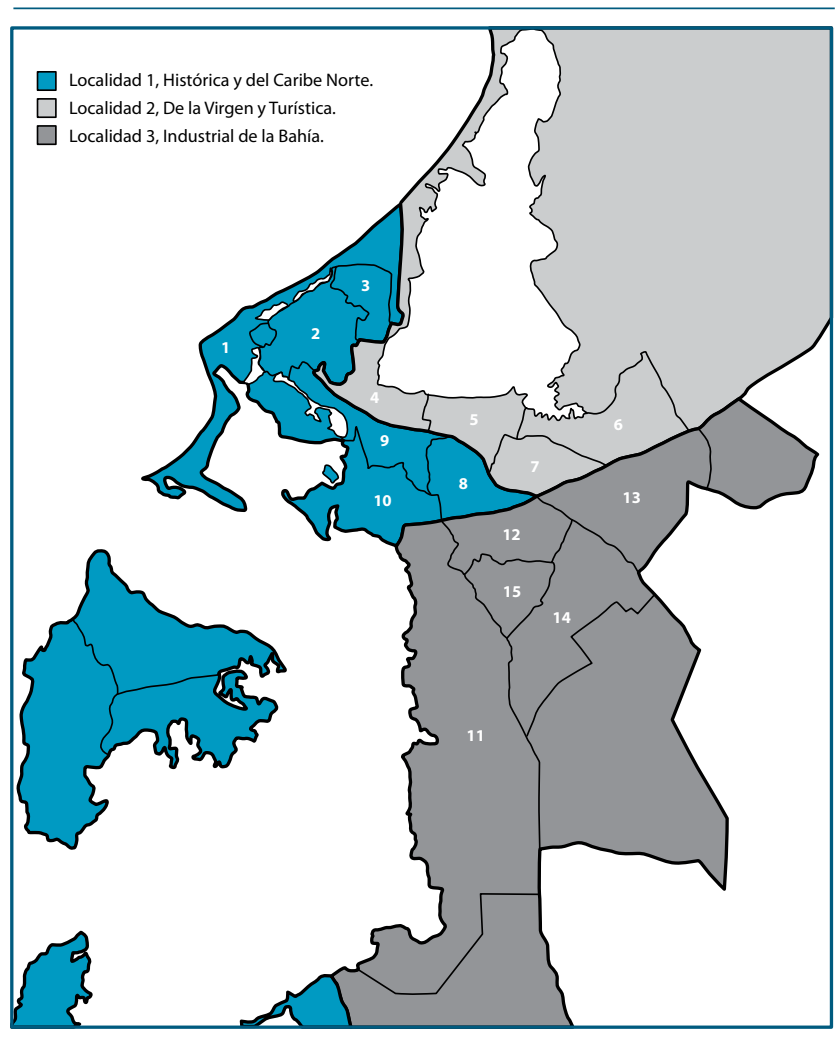

Masa Corporal $(\mathrm{IMC})=$ peso $(\mathrm{kg}) /$ talla, al cuadrado $\left(\mathrm{m}^{2}\right)^{(21)} \mathrm{y}$ el porcentaje de masa grasa medido a través de bioimpedancia eléctrica, tomando como referencia los valores de diagnóstico propuestos por la OMS (tabla 2 y 3 ).

Tabla 2. Valores de referencia IMC

\begin{tabular}{l|c|c} 
Clasificación & \multicolumn{2}{|c}{ IMC (Kg/m2) } \\
& Valores principales & Valores adicionales \\
\hline Sobrepeso & $\geq 25.00$ & $\geq 25.00$ \\
\hline Obesidad & $\geq 30.00$ & $\geq 30.00$ \\
\hline
\end{tabular}

Fuente: OMS/OPS 2016

Tabla 3. Valores de referencia porcentaje (\%) de grasa corporal

\begin{tabular}{c|c} 
Mujeres & PGC(\% de grasa corporal) \\
\hline Más de $30 \%$ & Exceso de grasa corporal \\
\hline Hombres & PGC(\% de grasa corporal) \\
\hline Más del $20 \%$ & Exceso de grasa corporal \\
\hline
\end{tabular}

Fuente: OMS/OPS 2016

Los instrumentos usados para la toma de medidas antropométricas siguieron las recomendaciones de las guías de atención de la obesidad del Ministerio de Salud colombiano, 
para medir el peso se utilizaron balanzas de pie digitales Marca TEZZIO TB-30037 con una capacidad de 120 kg máximo y una precisión de $100 \mathrm{~g}$; para la toma de la talla se utilizaron tallímetros portátiles con una capacidad de dos metros y una sensibilidad de $1 \mathrm{~mm}$.

Para la toma de la bioimpedancia eléctrica se utilizaron bioimpedanciómetros marca ONROM. Para medir este indicador, la temperatura ambiental fue de $20^{\circ} \mathrm{C}$ a $35^{\circ} \mathrm{C}$; además, se pidió a los pacientes, tener en cuenta las recomendaciones siguientes: no usar diuréticos por una semana, no beber alcohol en las 48 horas antes del examen, no realizar el ejercicio intenso por lo menos 12 horas antes, no ingerir alimentos ni bebidas cuatro horas antes. Se requería, por otra parte, vaciar la vejiga antes del análisis, no utilizar objetos metálicos y no realizar esta prueba a personas con marcapasos o en estado de embarazo.

Por otro lado, se realizó una encuesta de frecuencia de consumo para determinar la relación entre los participantes que consumen (expuestos) y no consumen edulcorantes no calóricos (no expuestos), considerando la frecuencia de consumo de cualquier producto endulzado con edulcorante no calórico o la utilización genérica del mismo en su dieta y otras variables relacionadas con el consumo, como la marca y el conocimiento del componente, entre otras.

\section{Análisis de los datos}

Para el análisis de los datos sobre el estado de salud y enfermedad ( $\mathrm{IMC} \geq 25$ ) de los participantes se utilizó la fórmula de "prevalencia puntual". Como medida de asociación (estimador de riesgo) se utilizó la "razón de prevalencias" (nivel de confianza de 0,95 ), ya que la exposición al factor (consumo diario de edulcorantes no calóricos) y enfermedad (IMC $\geq 25$ ) correspondió a un momento concreto del tiempo. Las fuentes de datos se analizaron con el programa estadístico WinEpi.

\section{Aspectos éticos}

Los participantes fueron seleccionados aleatoriamente y, previo al inicio del estudio, se explicaron claramente los objetivos, pidiéndose posteriormente, los participantes firmaron un consentimiento informado previamente avalado por el comité de bioética de la Universidad del Sinú, seccional Cartagena, para participar en el estudio.

\section{Resultados}

Los datos fueron recogidos en la unidad comunera de gobierno No. 1 de la ciudad de Cartagena de Indias. Se visitaron 88 viviendas en una totalidad de 12 barrios (tabla 4).

Las características sociodemográficas de la población estudiada se muestran en la tabla 5.
Tabla 4 Participantes en el estudio

\begin{tabular}{c|c|c}
$\begin{array}{c}\text { Unidad } \\
\text { Comunera de } \\
\text { Gobierno }\end{array}$ & Barrios & $\begin{array}{c}\text { Número de } \\
\text { viviendas } \\
\text { visitadas }\end{array}$ \\
\hline Unidad 1 & $\begin{array}{c}\text { El Laguito, Castillo } \\
\text { Grande, Bocagrande, } \\
\text { El Centro, La matuna, } \\
\text { Getsemaní, San Diego, } \\
\text { El cabrero, Marbella, } \\
\text { Crespo Chambacú, Pie } \\
\text { de la Popa y Manga }\end{array}$ & \\
\hline
\end{tabular}

* Los barrios la Matuna y Chambacú fueron excluidos del estudio ya que su estratificación es menor a 4.

Tabla 5. Características sociodemográficas de la población estudiada

\begin{tabular}{l|c|c|c}
\hline \multicolumn{1}{|c|}{ Variables } & Edad (Medias y DS) & N & $\%$ \\
\hline Sexo & & & \\
Masculino & $28 \pm 7$ & 190 & 40,43 \\
Femenino & $29 \pm 9$ & 280 & 59,57 \\
\hline
\end{tabular}

De acuerdo al análisis de prevalencias puntuales de la enfermedad (IMC $\geq 25$ ) se encontró una prevalencia de sobrepeso (IMC $\geq 25$ ) del 34\%, una prevalencia de Obesidad (IMC $>30$ ) del $9 \%$ y una prevalencia de porcentaje $(>20 \%)$ de grasa corporal aumentado del $38 \%$.

Las frecuencias de las variables del estudio observadas y esperadas, utilizando un nivel de confianza de 0,95 , se muestran en la tabla 6.

Tabla 6. Frecuencias observadas y esperadas (nivel de confianza del 95\%)

\begin{tabular}{|c|c|c|c|c|}
\hline \multicolumn{5}{|c|}{ Frecuencias observadas } \\
\hline & & \multicolumn{2}{|c|}{ Variable de riesgo } & \multirow[b]{2}{*}{ Total } \\
\hline & & $\begin{array}{c}\text { Expuestos } \\
130\end{array}$ & $\begin{array}{c}\text { No expuestos } \\
340\end{array}$ & \\
\hline \multirow{2}{*}{$\begin{array}{l}\text { Estado de } \\
\text { salud }\end{array}$} & Enfermos & 10 & 190 & 200 \\
\hline & Sanos & 120 & 150 & 270 \\
\hline & Total & 130 & 340 & 470 \\
\hline \multicolumn{5}{|c|}{ Frecuencias esperadas } \\
\hline & & \multicolumn{2}{|c|}{ Variable de riesgo } & \multirow[b]{2}{*}{ Total } \\
\hline & & $\begin{array}{c}\text { Expuestos } \\
130\end{array}$ & $\begin{array}{c}\text { No expuestos } \\
340\end{array}$ & \\
\hline \multirow{2}{*}{$\begin{array}{l}\text { Estado de } \\
\text { salud }\end{array}$} & Enfermos & 55,32 & 144,68 & 200 \\
\hline & Sanos & 74,68 & 195,32 & 270 \\
\hline \multicolumn{2}{|r|}{ Total } & 130 & 340 & 470 \\
\hline
\end{tabular}


En cuanto a la estimación del riesgo entre enfermos (exceso de peso (IMC $\geq 25$ )) y expuestos al consumo de edulcorantes no calóricos diario en la dieta, se obtuvieron los resultados relacionados en la tabla 7.

Tabla 7. Estimación del riesgo por razón de prevalencias de los participantes expuestos y no expuestos al consumo de edulcorante diario frente al desarrollo de la enfermedad (IMC $\geq 25$ )

\begin{tabular}{l|c}
\hline Significación & $\begin{array}{c}\text { Resultado significativo } \\
(0,0753,0,2516) \\
\text { Aproximación logarítmica: IC 0,959 } \\
\text { Aproximación Chi²: IC 0,950 }\end{array}$ \\
\hline Razón de prevalencias & $\mathbf{0 , 1 3 7 7}$ \\
\hline Resultados adicionales & \\
Prevalencia entre expuestos (130) & 0,0769 \\
Prevalencia entre no expuestos (340) & 0,5588 \\
\hline
\end{tabular}

De acuerdo con estos datos, los individuos expuestos al uso diario de edulcorantes no calóricos (130) presentaron entre 3,98 y 13,28 menos probabilidades de estar enfermos (IMC $\geq 25$ ) que los no expuestos al uso de edulcorantes no calóricos diariamente (340) (usando los límites de la aproximación logarítmica).

\section{Discusión}

La idea de implementar estrategias innovadoras de promoción de "factores protectores" de la salud, como se comentó anteriormente, es promover estos hábitos, conductas o estilos de alimentación adecuados y saludables de forma "voluntaria", aumentando la alimentación inteligente o racional y restringiendo el uso de conductas negativas, dañinas para la salud. Ahora es preciso resaltar que un momento ideal para hacer uso de este recurso sería en las etapas de la infancia y la adolescencia, fortaleciendo los estilos de vida saludables que pueden permanecer por el resto de la vida adulta $^{(31,32)}$.

Es el caso de México, donde fue establecido un acuerdo Nacional para la Salud Alimentaria que involucra las organizaciones públicas y privadas y la sociedad, para combatir la epidemia de obesidad a través del cumplimiento de 10 objetivos prioritarios, dentro de los cuales uno tiene que ver con la disminuir en el consumo de azúcares y otros edulcorantes calóricos añadidos en los alimentos, entre otros, aumentando la disponibilidad y accesibilidad de alimentos reducidos o con edulcorantes no calóricos añadidos ${ }^{(33)}$.

Sin embargo, la promoción de "edulcorantes" en la dieta diaria ha sido objeto de múltiples polémicas por lo que respecta a su seguridad a largo plazo; por esta razón, la forma más adecuada de enfocar esta polémica es desde la perspectiva del balance riesgo-beneficio ${ }^{(34)}$.
En Estados Unidos, la FDA vetó la utilización del "ciclamato sódico" en alimentos, tras un estudio que concluyó que el edulcorante artificial podría ser cancerígeno si se consume en cantidades muy elevadas ${ }^{(35)}$. Ese mismo año, Inglaterra, Bélgica, Irlanda y Nueva Zelanda también vetaron su uso. Recientemente, México, Chile, Argentina y Venezuela recomendaron a productores de alimentos utilizar otros edulcorantes diferentes al ciclamato para la preparación de bebidas dietéticas. Sin embargo, otros estudios determinaron que el edulcorante es "seguro" si no se consume en cantidades anormales desestimando los resultados en animales.

Finalmente, aparece otro tema de discusión entre los investigadores y es el efecto que pueda darse en una sociedad en la que se ingieren cada vez más "alimentos industrializados" poco nutritivos, como los productos endulzados con edulcorantes artificiales ello podría llevarnos, a largo plazo, al polo opuesto de la enfermedad.

\section{Conclusiones}

De acuerdo con los resultados del estudio se podría considerar que el uso diario de edulcorantes no calóricos en la dieta de los participantes fue un "factor protector" asociado al exceso de peso.

Sin embargo, a pesar de que el diseño transversal del estudio es adecuado para la estimación de prevalencias y algunas asociaciones, una debilidad consiste en que no es apropiado para el establecimiento de relaciones de causa-efecto, por lo que objetivos más ambiciosos requerirán de estudios longitudinales y muestras de tamaños mayores.

Por otro lado, a pesar de que la encuesta del consumo de edulcorantes mostró ser un instrumento adecuado, es importante destacar las diferencias entre este método y los utilizados en otros estudios que buscaban, a diferencia del nuestro, una estimación de la cantidad de consumo del edulcorante.

Como conclusión final, es el consumidor quien debe decidir si asume en algunos casos un riesgo muy remoto como contrapartida de las ventajas que le reporta el uso de determinados productos que en este caso, serían la reducción de las calorías ingeridas sin renunciar a determinados alimentos o sabores, además de otros efectos beneficiosos sobre el organismo, especialmente en la prevención de los trastornos cardiovasculares y de ciertos procesos tumorales ${ }^{(36)}$.

Aunque este efecto preventivo se produce fundamentalmente con la reducción del contenido de la grasa de la dieta, la limitación del consumo de azúcares, además de disminuir el aumento de tamaño del tejido adiposo, está relacionada con la disminución de la resistencia a la leptina causante de la baja sensación de saciedad y, en este caso, los edulcorantes artificiales serían de gran ayuda ${ }^{(37)}$. 


\section{Referencias}

1. Martinez, Rocio Robledo; DÍAZ, Fabio Alberto Escobar. Las enfermedades crónicas no transmisibles en Colombia. Boletín del observatorio en salud, 2010, vol. 3, no 4 .

2. De Pediatría, Sociedad Argentina; SUBCOMISIONES, Comités. Guías de práctica clínica para la prevención, el diagnóstico y el tratamiento de la obesidad. Arch argent pediatr, 2011, vol. 109, no 3, p. 256-266.

3. Peña, Manuel; Bacallao, Jorge. La obesidad en la pobreza: un problema emergente en las Américas. La obesidad en la pobreza: un nuevo reto para la salud pública. Washington, DC: Organización Panamericana de la Salud, 2000, p. 3-11.

4. González Jiménez, Emilio, et al. Prevalencia de sobrepeso y obesidad nutricional e hipertensión arterial y su relación con indicadores antropométricos en una población de escolares de Granada y su provincia. Nutrición Hospitalaria, 2011, vol. 26, no 5, p. 1004-1010.

5. Braguinsky, J. Prevalencia de obesidad en América Latina. En Anales del sistema Sanitario de Navarra. 2002. p. 109-115.

6. Gurruchaga, Alberto Maiz. Consecuencias patológicas de la obesidad: hipertensión arterial, diabetes mellitus y dislipidemia. Boletín Escuela de Medicina. Pontificia Universidad Católica de Chile, 1997, vol. 26, no 1, p. 18-2.

7. Blanco, Vanessa et al. La obesidad como problema de salud pública y su representación en el periódico el tiempo del 2007 al 2009. Trabajo de Grado. Universidad Javeriana. Repositorio 2009.

8. Martinez, Rocio Robledo; Díaz, Fabio Alberto Escobar. Las enfermedades crónicas no transmisibles en Colombia. Boletín del observatorio en salud, 2010, vol. 3, no 4.

9. Rangel Caballero, Luis Gabriel; Rojas Sánchez, Lyda Zoraya; Gamboa Delgado, Edna Magaly. Sobrepeso y obesidad en estudiantes universitarios colombianos y su asociación con la actividad física. Nutricion Hospitalaria, 2015, vol. 31, no 2.

10. Romero, Dario Mendoza; Urbina, Adriana. Actividad física en el tiempo libre y autopercepción del estado de salud en Colombia. Apunts. Medicina de l'Esport, 2013, vol. 48, no 177, p. 3-9.

11. Ministerio de la Protección Social, Instituto Nacional de Salud, Instituto Colombiano de Bienestar Familiar. Encuesta Nacional de la Situación Nutricional en Colombia (ENSIN) 2010. 2010

12. Cid, Patricia; Merino, José Manuel; Stiepovich, Jasna. Factores biológicos y psicosociales predictores del estilo de vida promotor de salud. Revista médica de Chile, 2006, vol. 134, no 12, p. 1491-1499.

13. El Ferrol, Arquitecto Marcide; Coruña, A. El paciente con exceso de peso: guía práctica de actuación en Atención Primaria. Rev Esp Obes, 2006, vol. 4, no 1, p. 33-44.

14. Basulto, J., et al. Análisis de un programa de pérdida de peso con sustitutivos de comidas sobre el control del peso y de parámetros bioquímicos en pacientes con sobrepeso y obesidad grado I. Nutrición Hospitalaria, 2008, vol. 23 , no 4, p. 388-394.

15. Encuesta Nacional de Consumo Alimentario (chile). Informe Final Ministerio de Salud de Chile.2010

16. Mayor, M.; Arillo, A.; Tiberio, G. Lipomatosis simétrica múltiple: a propósito de un caso. En Anales del Sistema Sanitario de Navarra. Gobierno de Navarra. Departamento de Salud, 2006. p. 433-437.

17. Campos Salazar, Cinthya; Solera Herrera, Andrea. The effect of different sport and physical activity courses on self-efficacy of costa rican university students. Actualidades Investigativas en Educación, 2013, vol. 13, no 3, p. 01-17.
18. Montoya Montoya, Susana; Múnera García, Nora Elena. Efecto de la intervención nutricional temprana en el resultado clínico de pacientes en riesgo nutricional. Nutrición Hospitalaria, 2014, vol. 29, no 2, p. 427-436.

19. Peña, Manuel; Bacallao, Jorge. La obesidad en la pobreza: un problema emergente en las Américas. La obesidad en la pobreza: un nuevo reto para la salud pública. Washington, DC: Organización Panamericana de la Salud, 2000, p. 3-11.c

20. Hano García, Olga Marina; Wood Rodríguez, Lisette; Villa Jiménez, Oscar Manuel. Obesidad y riesgo de cáncer colorrectal. Revista Cubana de Investigaciones Biomédicas, 2011, vol. 30, no 2, p. 251-259.

21. Puche, Rodolfo C. El índice de masa corporal y los razonamientos de un astrónomo. Medicina (Buenos Aires), 2005, vol. 65, no 4, p. 361-365.

22. Gutiérrez, Pedro Monterrey; Maury, Carmen Porrata. Procedimiento gráfico para la evaluación del estado nutricional de los adultos según el índice de masa corporal. Rev Cubana Aliment Nutr, 2001, vol. 15, no 1, p. 62-7.

23. Hernández Triana, Manuel; Ruiz Álvarez, Vladimir. Obesidad, una epidemia mundial: Implicaciones de la genética. Revista Cubana de Investigaciones Biomédicas, 2007, vol. 26, no 3, p. 0-0.

24. Stunkard, Albert J. Factores determinantes de la obesidad: opinión actual. La obesidade en la pobreza: un nuevo reto para la salud pública. Washington DC: Organización Panamericana de la Salud, 2000, p. 27-32.

25. Barceló Acosta, Malicela; Borroto Díaz, Gerardo. Estilo de vida: factor culminante en la aparición y el tratamiento de la obesidad. Revista Cubana de Investigaciones Biomédicas, 2001, vol. 20, no 4, p. 287-295.

26. Osorio, Alexandra Giraldo, et al. La promoción de la salud como estrategia para el fomento de estilos de vida saludables. Revista Hacia la Promoción de la Salud, 2010, vol. 15, no 1, p. 128-143.

27. Giannuzzi, Leda; Molina Ortiz, S. Edulcorantes natural y sintético: Aplicaciones y aspectos toxicológicos. Acta Farm. Bonaerense, 1995, vol. 14, no 2, p. $119-133$

28. García-Almeida, J. M.; Casado Fdez, Gracia M.; García Alemán, J. Una visión global y actual de los edulcorantes: aspectos de regulación. Nutrición Hospitalaria, 2013, vol. 28, p. 17-31.

29. Martínez, José Antonio Cernuda; García, Andrea Fernández. Los edulcorantes y su papel sobre el metabolismo humano. RqR Enfermería Comunitaria, 2016, vol. 4, no 2, p. 13-22.

30. Martínez, José Antonio Cernuda; García, Andrea Fernández. Los edulcorantes y su papel sobre el metabolismo humano. RqR Enfermería Comunitaria, 2016, vol. 4, no 2, p. 13-22.

31. García García, Isabel. Promoción de la salud en el medio escolar. Revista española de Salud pública, 1998, vol. 72, no 4, p. 285-287.

32. Devís Devís, José; Peiró Velert, Carmen. La actividad física y la promoción de la salud en niños/as y jóvenes. Revista de psicología del deporte, 1993 vol. 2, no 2, p. 0071-86.

33. Córdova-Villalobos, J. A. El Acuerdo Nacional para la Salud Alimentaria como una estrategia contra el sobrepeso y la obesidad. Cir, 2010, vol. 78, p. $105-107$.

34. García-Almeida, J. M.; Casado Fdez, Gracia M.; García Alemán, J. Una visión global y actual de los edulcorantes: aspectos de regulación. Nutrición Hospitalaria, 2013, vol. 28, p. 17-31.

35. Giannuzzi, Leda; Molina Ortiz, S. Edulcorantes natural y sintético: Aplicaciones y aspectos toxicológicos. Acta Farm. Bonaerense, 1995, vol. 14, no 2, p. 119-133.

36. Chimenos Küstner, E. Aspectos prácticos en la prevención del cáncer oral. Avances en Odontoestomatología, 2008, vol. 24, no 1, p. 61-67.

37. Villaseñor, Alfonso. El papel de la leptina en el desarrollo de la obesidad. Revista de endocrinología y nutrición, 2002, vol. 10, no 3, p. 135-139. 